\section{COPD-Therapie mit geringer Persistenz und Adhärenz}

Mueller S et al. Non-persistence and non-adherence to long-acting COPD medication therapy: A retrospective cohort study based on large German claims dataset. Respir Med 2017; 122: $1-11$

Die Effektivität der COPD-Therapie mit langwirksamen Bronchodilatatoren im Alltag hängt wesentlich von guter Therapiekontinuität (Persistenz) und -implementierung (Adhärenz) ab. Allerdings sind sowohl NonPersistenz (NP) und Non-Adhärenz (NA) weit verbreitet. Sabrina Müller et al. untersuchten bei deutschen COPD-Patienten das Ausmaß der NP und NA.

Die retrospektive Kohortenanalyse basierte auf Daten der Allgemeinen Ortskrankenkasse Nordost (1,8 Millionen Versicherte) aus den Jahren 2010-2012. Die Auswertung umfasste Daten von kontinuierlich Versicherten, die 2010 2011 eine COPD-Diagnose hatten und mindestens 1 Verordnung für langwirksame COPD-Medikamente bekamen. Zusätzlich bildeten Personen, die 6 Monate vor erstmaliger Verordnung eines langwirksamen COPD-Medikaments keine entsprechende Verschreibung erhalten hatten, die Gruppe therapienaiver Patienten. Ausgeschlossen waren Patienten mit einer begleitenden Asthmadiagnose und mit einem Alter < 40 Jahre. In der therapienaiven Gruppe analysierte die Arbeitsgruppe die NP, definiert als Behandlungslücke von >90 Tage. Ein Medikamentenwechsel innerhalb derselben Wirkstoffklasse galt als Therapiefortführung, solange keine Behandlungslücke >90 Tage bestand. NA war definiert als Medikationsverfügbarkeitsverhältnis (MPR, Medication Possession
RATIO) $<80 \%$. Die Adhärenz wurde nur bei Patienten mit kontinuierlicher Einnahme langwirksamer Bronchodilatatoren bewertet.

\section{Ergebnisse}

Insgesamt 45-937 COPD-Patienten erhielten mindestens 1 Verordnung für langwirksame COPD-Medikamente. Darunter waren 22-276 neu behandelte und 13-841 therapienaive Patienten. Nach 12 Monaten erreichte die NP-Rate bei therapienaiven Patienten 65,3\%. Wirkstoffspezifisch ermittelten Müller et al. folgenden 12-Monats-NP-Raten:

- $58,5 \%$ für LABA

- 47,9\% für LAMA

- 78,0\% für inhalative Kortikosteroide (ICS)

- 69,4\% für LABA/ICS-Fixkombination

Die MPR über 12 Monate und alle Wirkstoffklassen betrug 57,9\% und der Anteil nicht adhärenter Patienten erreichte 70 $\%$. Während der Phasen kontinuierlicher Behandlung betrugen die MPR/NA-Raten:

- über alle Wirkstoffklassen 85,0

$\% / 30,1 \%$

- für LABA 89,3\%/28,2\%

- für LAMA 92,1\%/16,2\%

- für ICS 84,2\%/43,8\%

- für LABA/ICS-Fixkombination 84,1 $\% / 42,8 \%$

Zu den Faktoren, die mit einem frühzeitigen Therapieabbruch bzw. einer -unterbrechung assoziiert waren, gehörten u. a. weibliches Geschlecht, höherer Charlson-Komorbiditätsindex oder weniger Facharztbesuche. Im Vergleich zu einer initialen LABA-Behandlung (Referenz), war die Wahrscheinlich für NP bei Patienten, die mit einem LAMA starteten, geringer. Dagegen verstärkte ein Behandlungsbeginn mit ICS oder LABA/ICS-Fixkombination das Risiko für NP.

\section{FAZIT}

Die Untersuchung belegt für Deutschland niedrige Persistenz- und Adhärenzraten bei der COPD-Therapie mit langwirksamen Bronchodilatatoren aller Wirkstoffklassen. Etwa zwei Drittel aller neu behandelten Patienten führen ihre Behandlung nach 12 Monaten nicht fort und ein Drittel der Patienten mit Therapiekontinuität implementierten die Medikamenteneinnahme nicht ausreichend.

Matthias Manych, Berlin 